\title{
Ein Weihnachtsbrief Prof. Dr. Alexander Tschirchs, Bern, an seinen Bruder Prof. Dr. Otto Tschirch, Brandenburg a.d. Havel (1911)
}

\author{
Von Gottfried Schramm
}

Die wissenschaftliche Entwicklung der Pharmakognosie und Pharmazie ist weltweit und unabdingbar verbunden mit der Lehre und Forschung des 1856 zu Guben in der preußischen Niederlausitz geborenen, von 1890-1932 an der Universität Bern wirkenden «Altmeisters» der modernen Pharmakognosie und Professors der Pharmazeutischen Chemie, Alexander Tschirch, dessen Geburtstag sich am 17. Oktober 1986 zum 130. Male jährte.

Sein unermüdliches Eintreten für die pharmakognostische Lehre und Forschung prägte und beeinflußte weit über die Grenzen der Schweiz und Deutschlands hinaus damalige Entwicklungstrends pharmazeutischer Arbeitsrichtungen. Als Veröffentlichungen bio- und bibliographisehen Inhalts, die sein weitgespanntes Schaffen widerspiegeln, erschienen 1915 eine Zusammenstellung seiner «Vorträge und Reden», ${ }^{1} 1921$ seine Lebenserinnerungen «Erlebtes und Erstrebtes», ${ }^{2}$ wenig später «Fünfzig Jahre im Dienste der Pharmacie und Naturforschung (1872-1922)» ${ }^{3}$ und 1925/26 «Kongreßfahrten» ${ }^{4}$ und andere. Zudem ist seine "vita» fester Bestandteil mancher Biographien und Lexika, so. J. A. Häfligers «Biographikon bemerkenswerter Apotheker in der Schweiz» des Jahres $1943^{5}$ oder W.-H.Hein und H.-D. Schwarz' «Deutsche Apotheker Biographie» aus dem Jahre $1978^{6}$ und anderer Beiträge (s. Nekrolog A. Tschirchs ${ }^{7}$ ).

Dem Verfasser blieb es vorbehalten, seit 1968 wichtiges unveröffentlichtes biographisches Material zu «Leben und Werk» A. Tschirchs, vornehmlich aus seiner Berner Zeit, in einer Serie von Einzelbearbeitungen mit dem Ziel der Vorbereitung einer Gesamtbiographie vorzulegen ${ }^{8-23}$.

Im Sinne einer Dokumentation und Konservierung authentischer Zeugnisse pharmazeutischer Quellenforschung, sei es gestattet, nachfolgend einen Weihnachtsbrief A.Tschirchs des Jahres 1911 an seinen Bruder, den Historiker Professor Dr. Otto Tschirch (1858-1941) in Brandenburg an der Havel, wiederzugeben und zu kommentieren. 
Der auf persönlichem Briefbogen handschriftlich gefertigte Brief A. Tschirchs ist ein pharmaziehistorisches Zeitdokument und eröffnet als solches Einblick in das wissenschaftliche und private Geschehen.

\section{DR.A.TSCHIRCH}

Professor an der Universität

BERN

Lieber Bruder,

ich habe den letzten Abschnitt des Manuscriptes für den III Band meines Handbuches auf die Post gegeben - fühle mich frei wie eine Frau, die Zwillinge geboren hat und will die frohe Stimmung benutzen Dir und den Deinen meinen Weihnachtsgruß zu senden, obwohl die Ferien noch nicht begonnen haben und mancherlei sonstige Arbeit wartet.

Am Sonntag habe ich zum ersten Mal Beethovens Missa solemnis gehört u. zwar in einer, wie mir gesagt wurde, vorzüglichen Aufführung im Münster. Es war ein großer Genuß. Denn während ich zu den neueren Componisten kein rechtes Verhältnis finden kann, ist mir Beethoven immer noch vertraut und ich verstehe ihn so leidlich. Freilich im Gloria u. Credo war mir nicht alles klar. Aber Anni, die die Messe 4 oder 5 mal gehört hat (sie ging auch in die Proben) meinte, man müsse diese beiden Teile eben öfter hören.

Sonst gehen wir jetzt selten aus. Ich finde eine große Freude an diesem Leben «nach Innen», nachdem ich so lange «nach Außen» gelebt. Ich genieße jetzt sehr meine Familie, von der ich ja leider bald ein Glied verlieren werde und sitze dann um $1 / 29$ Uhr wieder am Schreibtisch. Da kommt man vorwärts. Morgen beginnen die Vorarbeiten für den IV Band.

Mein Vortrag in Dijon, den ich Dir deutsch sende, scheint meinen Fachgenossen gefallen zu haben. Er ist ins Französische, Ungarische, Italienische, Griechische und Rumänische übersetzt und wird auch noch ins Finnische, Holländische und Englische übersetzt werden - obwohl ich nicht finde, daß besonders viel an ihm daran ist. Aber ich kann, wenn jemand anfrägt ob er ihn übersetzen dürfe, nicht gut «nein» sagen. Besonders hat er in Frankreich gefallen. 
Mit Naundorfer hast Du wohl etwas Ärger. Ich las neulich in der Gartenlaube einen ziemlich dummen Angriff. Aber mit derlei muß man sich eben abfinden. Angriffe machen mich schon lange nicht mehr warm «Viel Feind, viel Ehr'». Ich muß mich jetzt auch wegen der Feige mit verschiedenen Botanikern herumschlagen. Aber noch sehe ich nirgends ein Loch in der Sache und Ascherson hat die «Urfeige» dadurch canonisiert, daß er sie in die Bibel der Systematiker, die Synopsis, aufgenommen hat. Ich habe eben verschiedenen «Coniferen» auf die Hühneraugen getreten und die schreien jetzt «au!»

Aber die werden sich schon beruhigen. Die Sache selbst ist eben doch ein wesentlicher Fortschritt in der Aufklärung des schwierigen Problems. Ich habe zahllose Briefe in der Angelegenheit erhalten, und bin auch z.Zt. mit Schweinfurth, dem Kenner der ägyptischen Feigen in einen Briefwechsel gerathen, der sich allerdings jetzt auf Mumien - Urnen und Gräberharze Ägyptens bezieht.

Es wird nämlich in einigen Wochen ein Buch eines meiner Schüler erscheinen, das ganz neue Einblicke in die chemische Natur der von den Alten benutzten Harze thun läßt. Dadurch daß der betr. Herr meine Harzuntersuchungsmethode auf die Jahrtausende alten Harze übertrug, ist er zu ganz überraschenden Ergebnissen gelangt. Wir haben aber ein halbes Dutzend Harze mit Sicherheit identifizieren können. Über einige Ergebnisse hat Maspero in der franz. Akademie berichtet, andere habe ich Inschan in Berlin, der mir Material sandte, übermittelt - es waren gegen 500 Analysen nöthig. Auch sonst fehlt es nicht an Arbeit und so will ich denn schließen.

Das Erdbeben neulich war hier ziemlich stark, wir erlebten es im Theater während der Aufführung der «Ratten». Es entstand eine Panik, die sich aber bald legte. Den meisten Radau machte der eiserne Vorhang, aber die oberen Ränge sollten doch stark geschwankt haben. Unser Haus hat nicht gelitten. Auch von den Tablaren ist nichts heruntergefallen.

Unser Theater ist jetzt sehr gut. Wir sahen endlich eine vorzügliche Aufführung des «Rosenkavalier», der mich sehr entzückte.

Mit herzlichen Weihnachtsgrüßen an Euch alle Dein getreuer A

Die Übertragung des vorliegenden Tschirch-Briefes vom 18. Dezember 1911 erfolgte unter Berücksichtigung der damals zeitgenössischen Orthographie. 
Dr. A. TSCHIRCH

Professor an der liniversitit

biliN

$$
15 / \sqrt{2} n
$$

Intur hisher

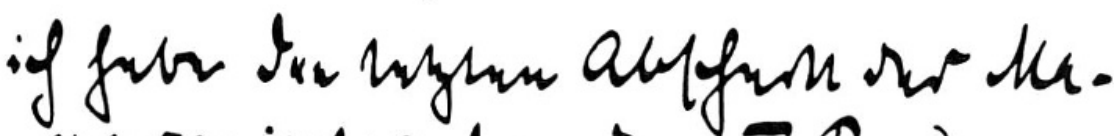

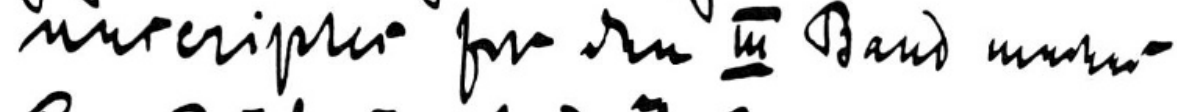

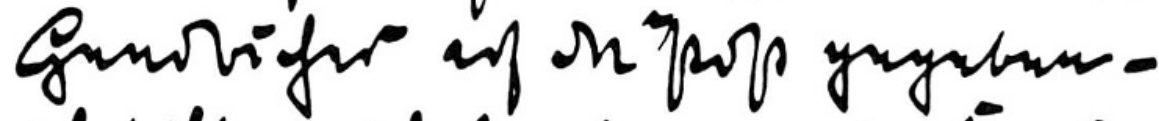
if foth ming fori now was frai

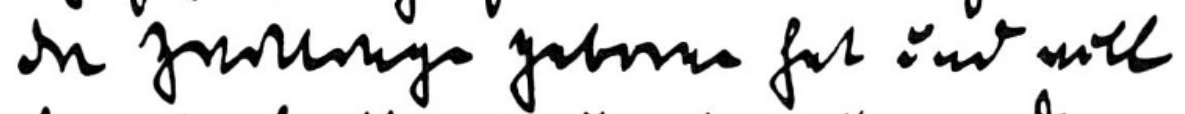

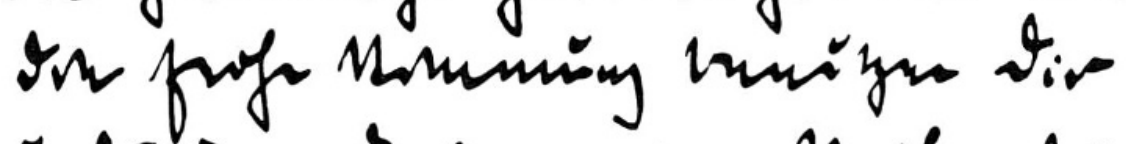

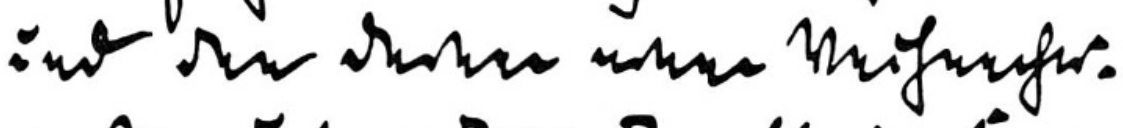
grip fo qunow, Onoufe on furs

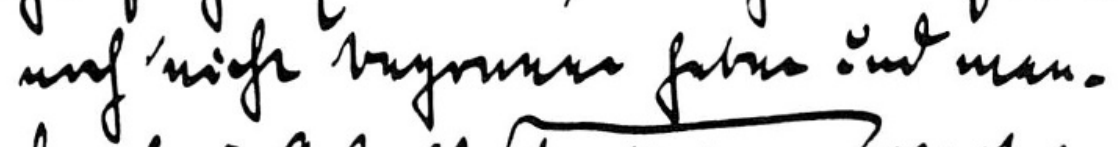

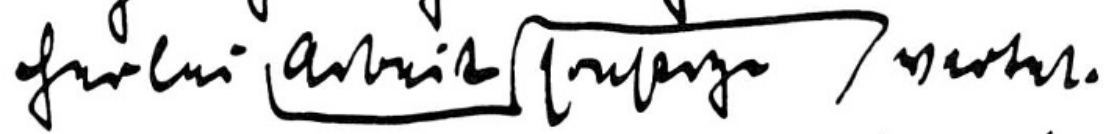

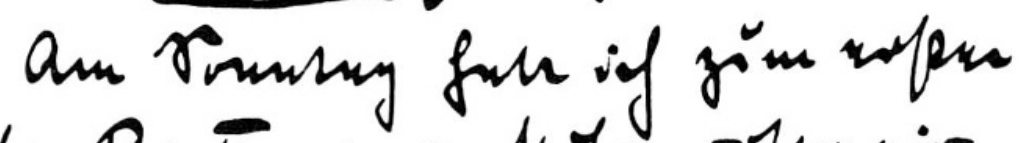

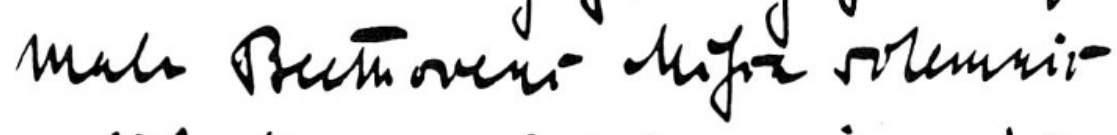

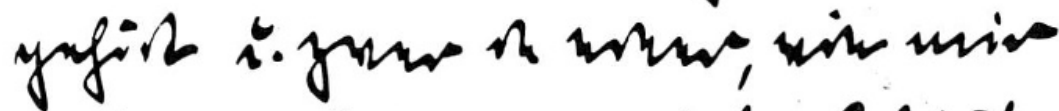

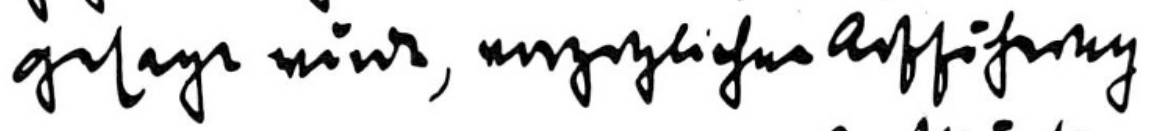
on Minfuna 


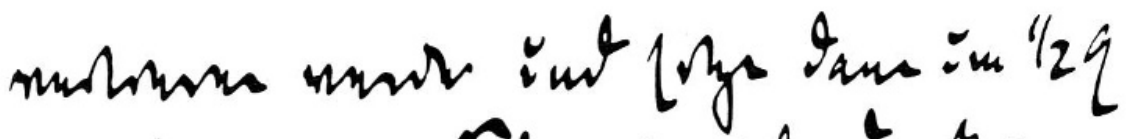

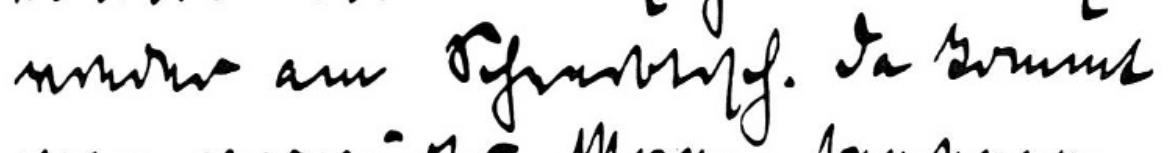
man surasiste. Mnyw bysume

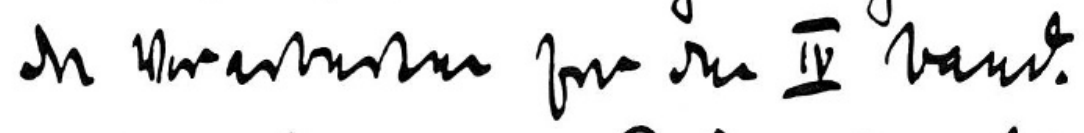
Wurk Notray a Digon, the if tis

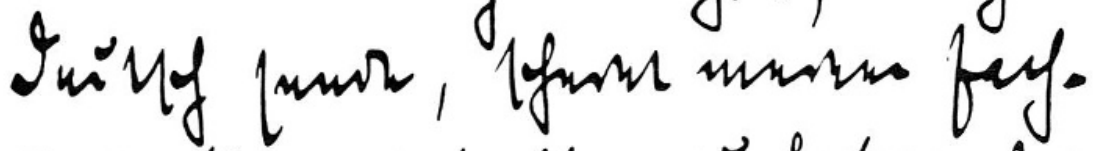

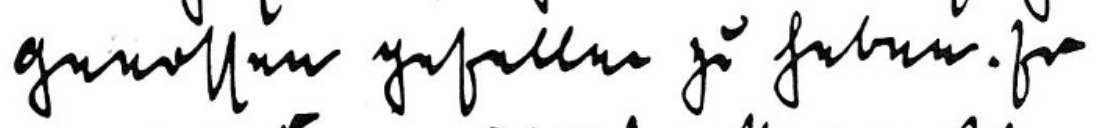
of no frangivifo, Huyarif,

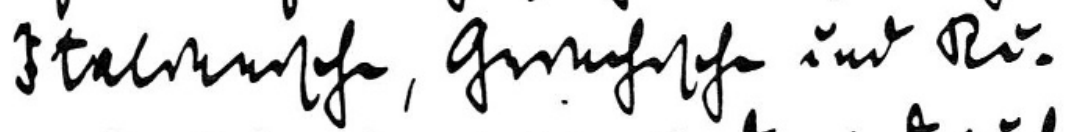

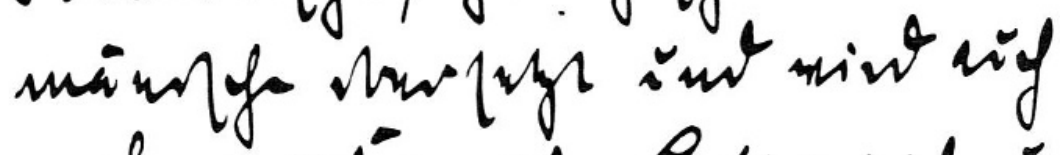

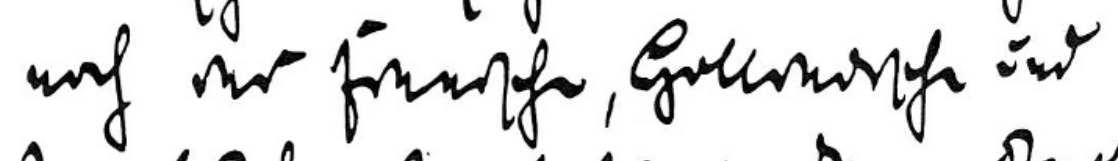

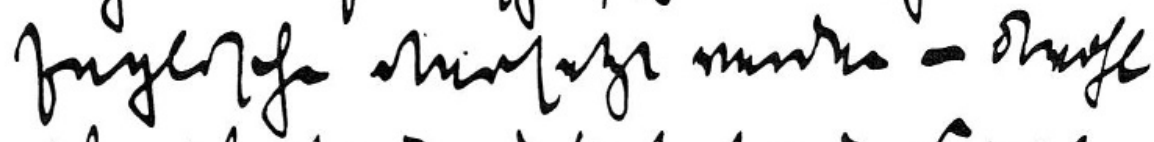

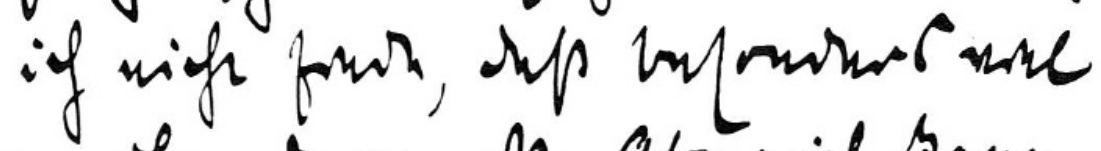
an ofm spor op. Abros if rawn, mun jumuno anposigh of us of

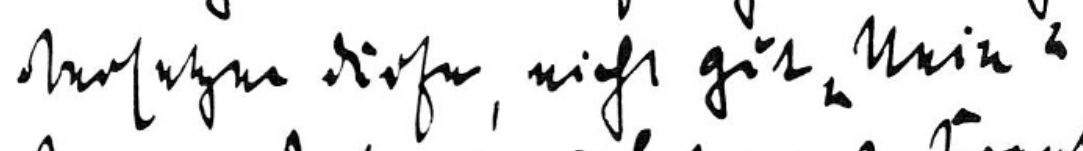

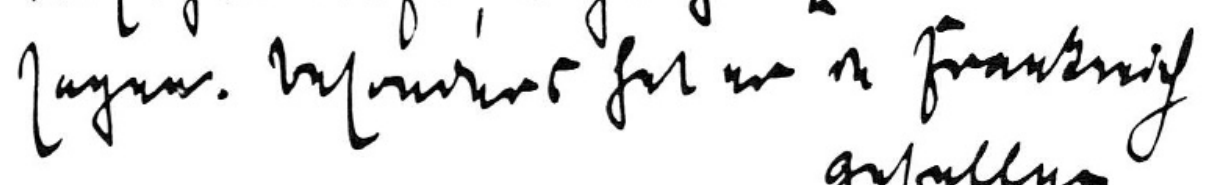
grallus

Textpassage zu A.Tschirchs Festvortrag in Dijon 1911 (dritte Seite)

479

Downloaded from Brill.com@4/26/2023 12:24: ๑7PM

via free access 


\section{Kommentar}

Der brieflich überlieferte Text zeigt A. Tschirch auf dem Höhepunkt seines Wirkens. Dem Biographen offenbart sich deutlich eine intensive Arbeitsphase an seinem umfassenden, dreibändigen und Lebenswerk, dem «Handbuch der Pharmakognosie», dessen Erscheinen für Bd.II/1 für die Jahre 1910-1912 nachweisbar ist. Darin wird die Pharmakognosie nicht mehr als Zweig der angewandten Botanik betrachtet, sondern als selbständige und etablierte Wissenschaft vorgestellt. Die botanischen Fragen wurden von den chemischen und physikalischen, die geographischen und historischen von den linguistischen und ethnographischen getrennt. Als pharmakognostische Einzeldisziplinen konzipierte A.Tschirch die Pharmakobotanik, die Pharmakochemie, die Pharmakogeographie, die Pharmakoethnographie und die Pharmakohistorie, wie dies auch in seinem noch heute lesenswerten Festvortrag anläßlich der Gründung der «Section des sciences pharmaceutiques» der «Association française pour l'avancement des sciences» in Dijon im Jahre 1911 zum Ausdruck kommt.

Als Forschungsarbeiten A.Tschirchs und seiner Schüler erschienen im Jahre 1911 zudem Studien über das «Feigenproblem», denen in späteren Jahren (1913 u. 1919) zwei Übersichtsarbeiten hierzu folgten.

Gedanklich naheliegend und historisch verständlich erscheint $A$. Tschirchs Kontakt zu dem pflanzengeographisch, botanisch, entwicklungsgeschichtlich und botanisch-physiologisch hervortretenden Paul Friedrich August Ascherson (1834-1913), dessen Hauptwerke in NUC pre-1956 imprints XXIII (1969), 275f. aufgeführt sind, sowie zu dem Forschungsreisenden Georg August Schweinfurth (1836-1925), dessen botanische sowie ethnologische Werke im gleichen Werk DXXXIII (1977), $70 \mathrm{f}$. genannt werden. 
Vom Rat der Stadt Brandenburg (DDR), erhalten wir mit Schreiben vom 30.1.1987 nachfolgende wichtige biographische Angaben über Professor Otto Tschirch:

«Geboren am 4.6.1858 als zweiter Sohn des Pfarrers Adolf Tschirch in Guben

1876-1880 Studium der Geschichte in Berlin

1882 Schuldienst zuerst in Berlin, dann im gleichen Jahr in Brandenburg an der Saldrina (Gymnasium), bis 1921 dort als Lehrer tätig

1884 Promotion

1899-1929 Stadtarchivar in Brandenburg

Gestorben am 13.3.1941 an den Folgen eines Verkehrsunfalls.»

\section{Literatur}

1 Schüler und Freunde [Hrsg.]: Vorträge und Reden von A.Tschirch, Leipzig 1915.

2 Tschirch, Alexander: Erlebtes und Erstrebtes. Lebenserinnungen, Bonn 1921.

3 Schweizerischer Apothekerverein [Hrsg.]: Fünfzig Jahre im Dienste der Pharmacie und Naturforschung (1872-1922), Zürich o.J.

4 Tschirch, [Alexander]: Kongreßfahrten, Wien 1925/26 [= Sonderabdruck aus der Pharmazeutischen Post].

5 Häfliger, Josef Anton: Biographikon bemerkenswerter Apotheker in der Schweiz, Basel 1943 [ = Separatabzug aus der Festschrift 100 Jahre Schweizerischer Apothekerverein], $292 \mathrm{f}$.

6 Hein, Wolfgang-Hagen u. Holm-Dieter Schwarz [Hrsg.]: Deutsche Apotheker-Biographie, Stuttgart 1978, Bd. II [ = Veröffentlichungen der Internationalen Gesellschaft für Geschichte der Pharmazie e. V.], $696 \mathrm{f}$.

7 Flück, H[ans]: Alexander Tschirch (1856-1939), in: Verhandlungen der Schweiz. Naturforschenden Gesellschaft [Locarno] 1940, 503-521.

8 Schramm, Gottfried: Ein Andenken an Alexander Tschirch, in: Deutsche ApothekerZeitung 108 (1968), 2027.

9 Ders.: Eine Glückwunschadresse der Schüler und Freunde Professor Dr.Alexander Tschirchs (1856-1939), Bern, zu dessen 70. Geburtstag am 17. Oktober 1926, in: Beiträge zur Geschichte der Pharmazie 28 (1976) [ = Beilage zur Deutschen Apotheker-Zeitung], 17-19.

10 Ders.: Professor Dr. Alexander Tschirch (1856-1939), Bern, als Pharmaziehistoriker, in: Pharmaceutica Acta Helvetica 52 (1977), 143-149.

11 Ders.: Kontakte Alexander Tschirchs und seiner Berner Schule zum Ausland (Beziehungen zu den Niederlanden) in: Schweizerische Apotheker-Zeitung 117 (1979), 558-561.

12 Ders.: Kontakte Alexander Tschirchs und seiner Berner Schule zum Ausland (K.H.Cederberg u. J. A. Edner), in: Schweizerische Apotheker-Zeitung 117 (1979), 611 f. 
13 Ders.: American Impressions of a Swiss Pharmacist (1894). An Unpublished Letter of F. A. Flückiger to A.Tschirch, in: Pharmacy in History 20 (1978), $111 \mathrm{f}$.

14 Ders.: Amerika-Impressionen eines Schweizer Pharmakognosten. Ein unbekannter Brief Friedrich August Flückigers (1828-1894) an Alexander Tschirch (1856-1939) vom 17. August 1894, in: Beiträge zur Geschichte der Pharmazie 31 (1978) [= Beilage zur Deutschen Apotheker Zeitung], $6 \mathrm{f}$.

15 Ders. mit Hertha Schramm: Ein Brief Alexander Tschirchs (1856-1939), Bern, an Kurt Siegfried (1873-1945), Zofingen, zur arzneilichen Bewertung von Digitalis ambigua Murray, in: Gottfried Schramm [Hrsg.]: Neue Beiträge zur Geschichte der Pharmazie. Festschrift für H.-R. Fehlmann zum 60. Geburtstag, Zürich, 1979, 179-188.

16 Ders.: Kontakte Alexander Tschirchs und seiner Berner Schule zum Ausland (Doktoranden aus Russisch Polen und dem Zaristischen Russland), in: Pharmazeutische Zeitung 125 (1980), 1674f.

17 Ders.: Kontakte Alexander Tschirchs und seiner Berner Schule zum Ausland (Ergänzende Daten und Fakten über Doktoranden aus Polen), in: Schweizerische ApothekerZeitung 118 (1980), 494-496.

18 Ders.: Ein unbekannter Brief von Carlo de Marchesetti (1850-1926), Triest, an Alexander Tschirch (1856-1939), Bern, in Österreichische Apotheker-Zeitung 120 (1980), $983 \mathrm{f}$.

19 Ders.: Ein Brief von Hermann Thoms an Alexander Tschirch, in: Deutsche ApothekerZeitung $120(1980), 1267 \mathrm{f}$.

20 Ders.: Zwei Briefe von Hermann Schelenz (1848-1922), Cassel, an Alexander Tschirch (1856-1939), Bern, in: Pharmazeutische Zeitung 125 (1980), 408-413.

21 Ders.: Alexander Tschirch. Seine Doktoranden aus Deutschland in den Jahren 1889-1931, in: Schweizerische Apotheker-Zeitung 119 (1981), 51-55.

22 Ders.: Alexander Tschirch an seinen Bruder Otto Tschirch, in: Schweizerische Apotheker-Zeitung 119 (1981), 555 a f.

23 Ders.: Alexander Tschirch (1856-1939) and copper, in: Pharmacy International, November $1982,352 \mathrm{f}$.

PD Dr.sc.nat. Dr.phil. Gottfried Schramm, Chefapotheker Stadtspital Waid, Tièchestraße 99

CH-8037 Zürich 